\title{
A Rarity in Breast Disease: Metastatic Ovarian Carcinoma to the Breast Mimicking Inflammatory Breast Cancer
}

\section{Abstract}

Metastatic ovarian carcinoma to the breast has been documented in 110 cases with an incidence of $<1 \%$. Infrequently this can mimic inflammatory breast carcinoma making differentiation between breast and ovarian cancer challenging. Immunohistochemistry (IHC) markers like paired box gene 8 (PAX8) positivity, Wilms' tumor 1 (WT1) positivity, and gross cystic disease fluid protein 15 (GCDFP-15) negativity, however, can allow for diagnostic certainty. With only seven other cases of metastatic ovarian carcinoma mimicking inflammatory breast cancer, this case is an exceptional and unique find. The patient in this study presented with three months of left breast edema and erythema. Skin biopsies were performed to assess for inflammatory breast cancer. With her recent diagnosis of metastatic ovarian cancer to the breast, metastatic disease was high on the differential. The histopathology results and immunohistochemistry had confirmed the diagnosis.

Keywords: Metastatic ovarian cancer; Extra mammary metastasis; Inflammatory breast cancer

Received: June 05, 2017; Accepted: June 19, 2017; Published: June 25, 2017

\section{Introduction}

Based on statistics from the National Cancer Institute, the lifetime risk of ovarian cancer is approximately $1.3 \%$. By the time ovarian cancer is diagnosed, $60 \%$ of women have evidence of metastasis [1]. While the most common sites of metastasis are bowel, peritoneum, and omentum, metastasis to the breast occurs in $0.03-0.6 \%$ of cases. Even rarer is metastatic ovarian cancer presenting as inflammatory breast cancer with only seven other cases identified in the literature [2]. This report presents the eighth case of its kind.

\section{Case Report}

A 73-year-old woman presented to the office in March 2017 complaining of three months of redness, swelling and itchiness of the left breast. In October 2016, her mammogram and ultrasound were significant for $1.1 \mathrm{~cm}$ and $1.7 \mathrm{~cm}$ nodules in the left breast at the 3:00 and 4:00 positions, respectively, along with a $2.3 \mathrm{~cm}$ lymph node in the left axilla (Figure 1). Ultrasound guided biopsies of the two sites in the left breast as well as a fine needle aspiration biopsy of the left axillary lymph node revealed metastatic high grade serous carcinoma of ovarian origin. IHC had shown PAX8 and WT1 positivity with GCDFP-15 negativity. A

\section{Ben Fauzi El Attrache', Camryn Highsmith², Bradley Gluck ${ }^{3}$ Alan Heimann $n^{4}$ and Edna Kapenhas ${ }^{5}$}

\section{Department of Surgery at Southampton Hospital, NY, USA \\ 2 Junior at Southampton High School, NY, USA \\ 3 Department of Diagnostic Radiology at Southampton Hospital, NY, USA \\ 4 Department of Pathology at Stony Brook University Hospital NY, USA \\ 5 Department of Surgery at Southampton Hospital, NY, USA}

\section{Corresponding author: Edna Kapenhas}

ĐEKapenhas@southamptonhospital.org

Department of Surgery at Southampton Hospital Community Surgery Program: Southampton Hospital 240 Meeting House Lane, Southampton, NY 11968, USA.

Tel: $516-528-2130$

Citation: Attrache BFE, Highsmith C,Gluck B, et al. A Rarity in Breast Disease: Metastatic Ovarian Carcinoma to the Breast Mimicking Inflammatory Breast Cancer. J Univer Surg. 2017, 5:3.

follow up breast MRI in March 2017 had identified conglomerate masses spanning $11.8 \times 6.0 \times 8.2 \mathrm{~cm}$ in the left breast (Figure 2). Given the lack of response to a month of antibiotics for suspected mastitis, she was referred to the breast clinic for a skin biopsy to rule out inflammatory breast cancer.

Her past medical/surgical history was significant for a gastroesophageal reflux disease and a history of ovarian cancer with total abdominal hysterectomy, bilateral salpingooophorectomy, and omentectomy in 2011. There was a family history of breast cancer in her daughter at age 49. Breast cancer susceptibility gene (BRCA) testing was found to be negative. Physical examination revealed a swollen, tender, 


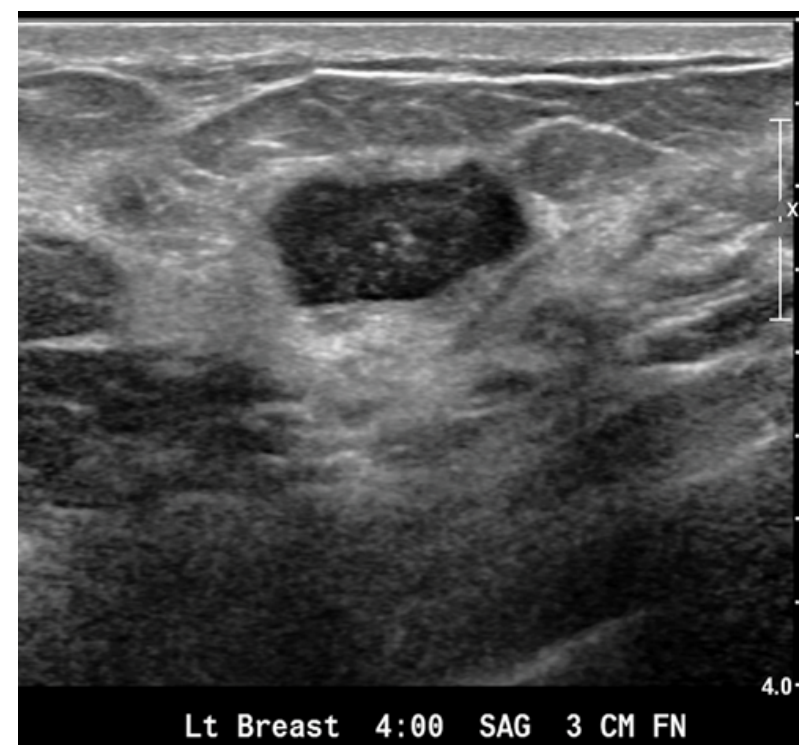

Figure 1 Left Breast sonogram showing a $1.7 \mathrm{~cm}$ mass in left breast at 4:00, $3 \mathrm{~cm}$ from the nipple.

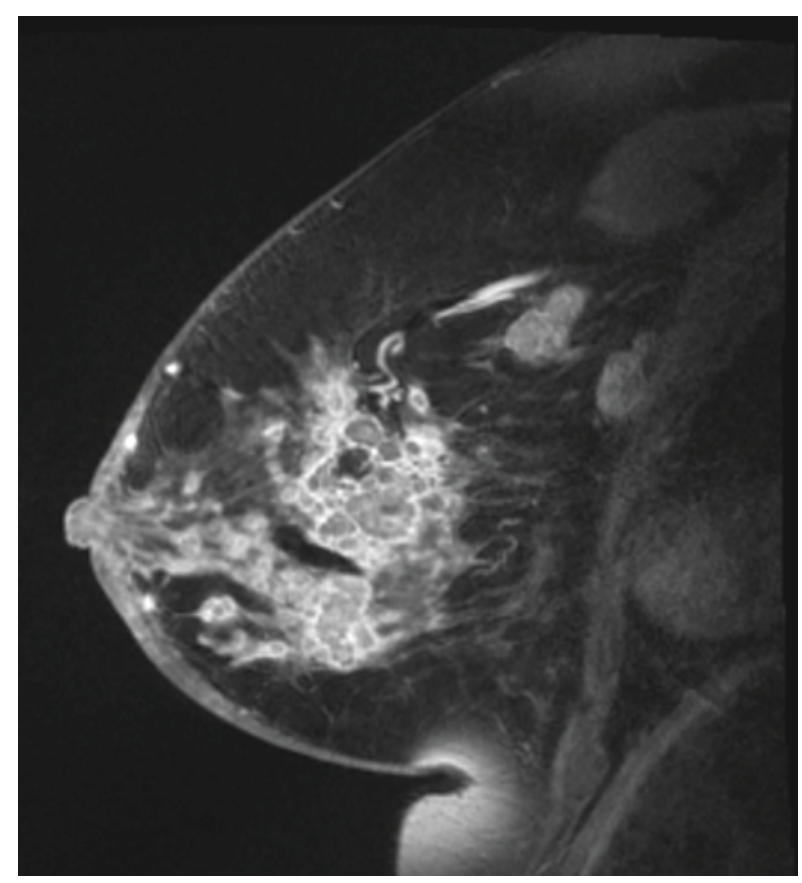

Figure 2 MRI Left Breast showing enhancement of the left outer breast consisting of multiple conglomerate masses with extension to the nipple areolar complex spanning $11.8 \times 6.0 \times 8.2 \mathrm{~cm}$. Skin thickening with edema is present. Enlargement of internal mammary vasculature is seen.

and erythematous left breast with thickened skin and edema. An approximately $12 \mathrm{~cm}$ palpable mass was noted in the lateral breast with extension medially towards the nipple, which was partially retracted. Punch biopsies were performed of two areas in the left breast which showed poorly differentiated invasive adenocarcinoma infiltrating the dermis (Figure 3) and involving dermal lymphatic's with PAX8 and WT1 positivity (Figures 4 and 5) and estrogen receptor (ER) and progesterone receptor (PR) negativity on IHC. This was consistent with ovarian cancer metastatic to the breast mimicking inflammatory breast cancer, clinically.

\section{Discussion}

Ovarian cancer metastasis occurs by hematogenous or lymphatic spread [3]. While its spread is primarily transperitoneal, advanced cases can spread to the liver, lung, and pleura [2]. Metastatic disease can also present in the breast and should be on the list of differential diagnoses, especially with an ovarian cancer history [3]. According to Tempfer et al. [4] a PubMed search revealed 110 total cases of metastatic ovarian cancer to the breast in February 2015 [4]. Overall, extramammary cancers metastasizing to the breast has an incidence of $0.1-1.3 \%$ [3].

DeLair et al. [5] had evaluated a group of 85 patients with nonmammary metastases to the breast and found that the most

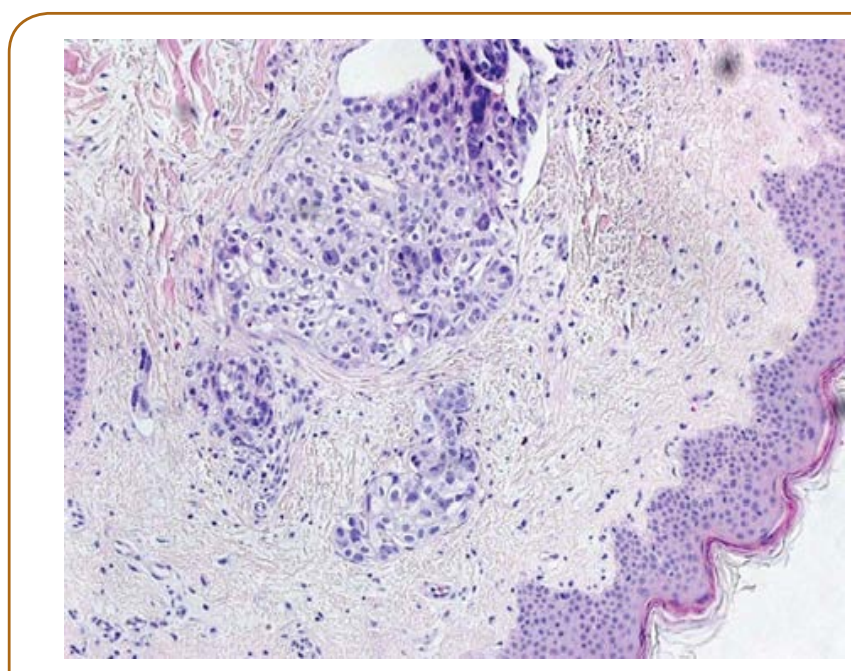

Figure 3 Skin with high grade serous carcinoma present in dilated lymphatics, Haemotoxylin \& Eosin (H\&E) Staining $100 \mathrm{X}$

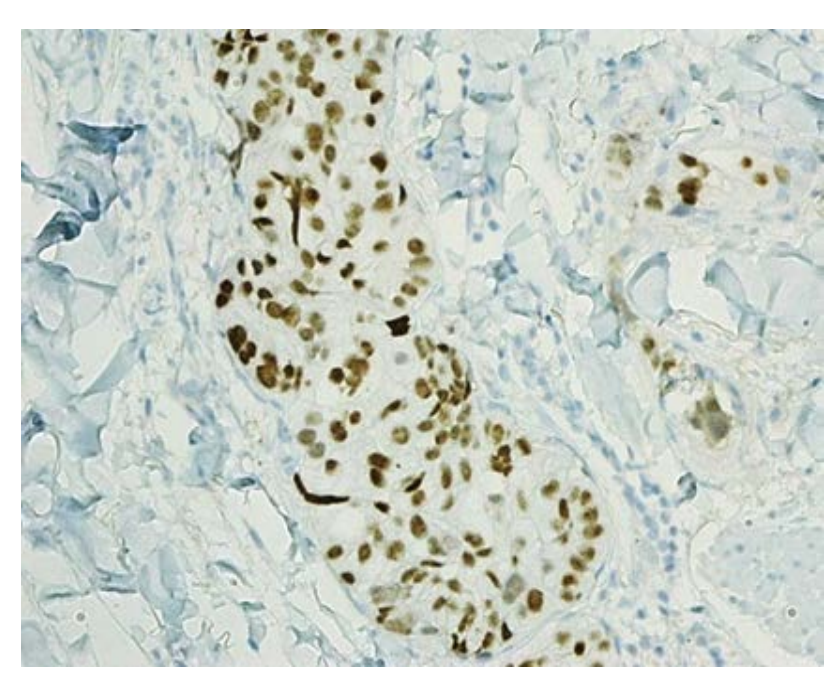

Figure 4 Dermal lymphatic IHC with anti-PAX8 stains tumor cell nuclei $200 \mathrm{X}$ 
common type of tumor was carcinoma with the most common subtype being serous ovarian carcinoma. Melanoma and sarcoma were the second and third most common, respectively. The features of breast cancer and ovarian metastasis tend to have overlapping IHC and morphology making the diagnosis difficult. However, certain markers like PAX8 were shown to be positive in ovarian carcinoma and negative in breast carcinoma. WT1, another marker, is positive in $85 \%$ of ovarian carcinomas with rare positivity (2.1\%) in breast cancer [5]. GCDFP-15 is a marker for breast differentiation that is $69 \%$ sensitive and $97 \%$ specific [2].

An important histological difference between ovarian and breast cancer is the type of calcifications. Ovarian has psammomatous calcifications whereas breast has non-psammomatous calcifications [5]. With the tumors having psammomatous calcifications and being WT1 positive, PAX 8 positive, and GCDFP-15 negative, this case was highly suggestive of ovarian carcinoma. Furthermore, the punch biopsy of the skin was also WT1 and PAX 8 positive, suggesting an ovarian source.

Inflammatory breast cancer is suspected based on the physical characteristics of erythema, nipple retraction, brawny induration,

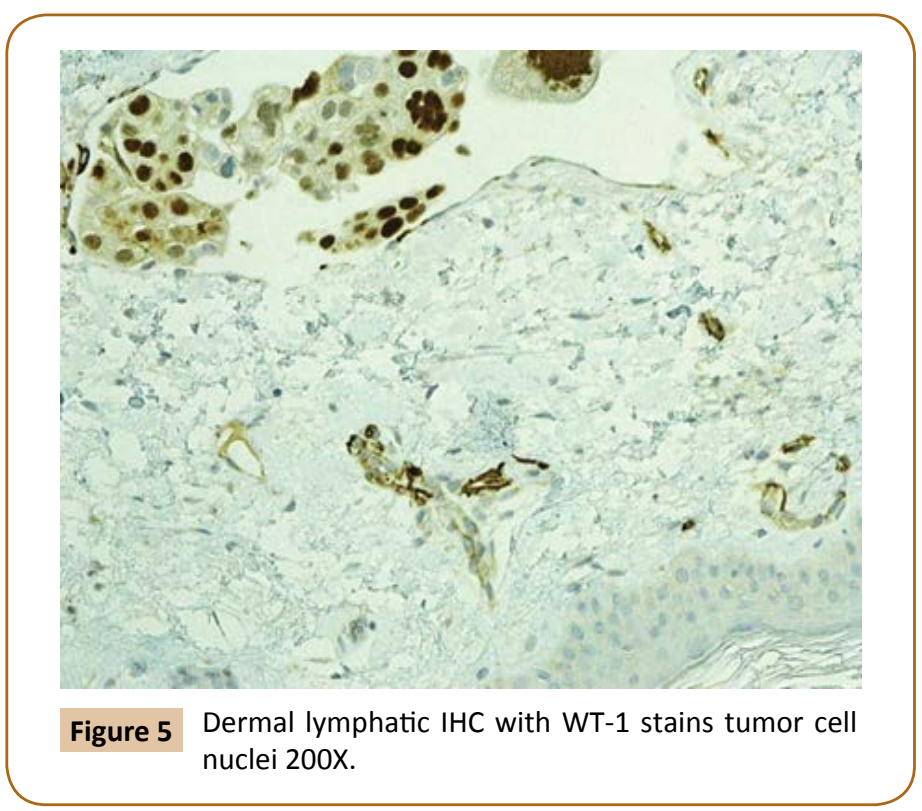

Table 1 Published cases of metastatic ovarian carcinoma to the breast mimicking inflammatory breast cancer.

\begin{tabular}{|r|c|c|c|}
\hline Case & Author & Year & \multicolumn{1}{c|}{ Ovarian Tumor } \\
\hline 1 & lbach & 1963 & Cyst-adenocarcinoma \\
\hline 2 & Krishnan & 1980 & Papillary Adenocarcinoma \\
\hline 3 & Ozguroglu & 1999 & Papillary Serous Adenocarcinoma \\
\hline 4 & Fondrinier & 1999 & Papillary Serous Adenocarcinoma \\
\hline 5 & Kayikcioglu & 2001 & Papillary Serous Adenocarcinoma \\
\hline 6 & Sato & 2007 & Clear Cell Carcinoma \\
\hline 7 & Klein & 2008 & Papillary Serous Adenocarcinoma \\
\hline
\end{tabular}

and edema (peau d'orange). The patients tend to present with axillary lymphadenopathy in greater than $75 \%$ of women. Inflammatory breast cancer occurs in $<3 \%$ of all breast cancers [6]. In rare cases, extra mammary cancers can clinically mimic inflammatory breast cancer. In regards to metastatic ovarian cancer presenting as inflammatory breast cancer, only seven other cases (Table 1) are documented and published [3].

The mainstay of treatment for metastatic ovarian cancer to the breast is chemotherapy. Surgery should not be considered for initial treatment [3]. Palliative surgery with mastectomy may be considered for those unresponsive to chemotherapy. Overall prognosis is poor with times ranging from 13 days to 3.5 years. One year survival is approximately $40 \%$. An even worse prognosis is conferred with inflammatory breast metastasis with times ranging from 3 to 18 months [7].

\section{Conclusion}

Metastatic ovarian cancer presenting as inflammatory breast cancer is a unique and infrequent diagnosis. It carries a grave prognosis, and therefore should be recognized as a differential in patients with history of ovarian cancer. Given the similarities between breast and ovarian cancer morphology, certain markers (PAX8, WT1, and GCDFP-15) assist in making a distinct diagnosis. Treatment focuses around systemic therapy with surgery only considered for palliative reasons.

\section{Conflict of Interest}

The authors have declared no conflicts of interest. 


\section{References}

1 SEER Cancer Stat Facts: Ovarian Cancer. National Cancer Institute. (2017)

2 Achariyapota V, Chuangsuwanich T, Benjapibal M (2016) Inflammatory breast cancer from metastatic ovarian Cancer. Case Rep Obstet Gynecol 1-3.

3 Amzeri M, Garcia C, Stanciu C, Veys I, Awada A, et al. (2014) Case Report: Mammary and rectal metastases from an ovarian cancer: Report of two cases and review of literature. F1000Res 3:1-8.

4 Tempfer CB, El Fizazi N, Ergonenc H, Solass W (2016) Metastasis of ovarian cancer to the breast: A report of two cases and a review of the literature. Oncol Lett 11: 4008-4012.

5 DeLair DF, Corben AD, Catalano JP, Vallejo CE, Brogi E, et al. (2013) Non-mammary metastases to the breast and axilla: a study of 85 cases. Mod Pathol 26:343-349.

6 Brunicardi CF, Andersen DK, Billiar TR, Dunn D, Hunter JG, et al. (2015). Schwartz's Principles of Surgery (10th ed.). New York, NY: McGraw-Hill Education.

7 Klein RL, Brown AR, Gomez-Castro CM, Chambers SK, Cragun JM, et al. (2010) Ovarian cancer metastatic to the breast presenting as inflammatory breast cancer: A case report and literature review. J Cancer 1:27-31. 